\title{
Cartas de Control Estadístico de Procesos en el Monitoreo de la Mortalidad Perinatal
}

\section{Statistical process control charts in perinatal mortality surveillance}

Nora E. Montoya-Restrepo ${ }^{1}$ y Juan C. Correa-Morales ${ }^{2}$

1 SUSALUD. Medellín, Colombia.noramore@susalud.com.co.

2 Escuela de Estadística, Universidad Nacional de Colombia, Sede Medellín. jccorrea@unalmed.edu.co

Recibido 27 Mayo 2008/Enviado para Modificación 29 Noviembre 2008/Aceptado 2 Enero 2009

\section{RESUMEN}

Objetivo Aplicar la metodología de las cartas de control estadístico de procesos (SPC) en la vigilancia de la mortalidad perinatal, indicador de la salud maternoperinatal.

Método Las cartas de control fueron elaboradas utilizando 286 muertes perinatales y los 51840 nacimientos, ocurridos en la Empresa Promotora de Salud SUSALUD, entre enero de 2004 y diciembre de 2007. Se elaboraron dos cartas de control, la primera se hizo teniendo en cuenta como línea central la proporción de muertes perinatales en cada uno de los meses y como límites superior e inferior de control la proporción de muertes $\pm 3 \mathrm{SD}$, la segunda se elaboró calculando los logits de las proporciones de las muertes perinatales.

Resultados Se construyeron dos cartas control para la vigilancia de la mortalidad perinatal. La primera tuvo en cuenta las proporciones de muertes perinatales para cada uno de los meses dando un promedio para el proceso de cinco muertes perinatales por cada mil nacimientos $(p=0,005)$, para la segunda carta control se calcularon los logits de las proporciones de muertes perinatales.

Conclusiones Disponer de las cartas SPC para el monitoreo y posterior análisis de la mortalidad perinatal, permitirá detectar rápidamente los cambios en la calidad del servicio, evaluar aspectos de la calidad de la atención materno-infantil y programar intervenciones específicas.

Palabras Clave: Vigilancia sanitaria, indicadores de salud, mortalidad perinatal (fuente: DeCS, BIREME).

\section{ABSTRACT}

Objective Applying statistical process control (SPC) charts in perinatal mortality surveillance (as an epidemiological indicator).

Method The control charts were prepared using 51,840 births; 286 cases were produced from these births. All information came from SUSALUD between January 2004 and December 2007. Two control charts are presented; the first one's central line was considered to be the proportion of cases $\pm 3 S D$ and the second one used the logits from the percentages of cases. 
Results Two control charts were prepared for monitoring perinatal mortality. The first considered the percentage of cases per month and an average of five cases per one thousand births was obtained $(p=0.005)$. The logits were used for the second chart.

Conclusions Having SPC charts available for monitoring and analysing perinatal mortality will allow changes in service quality to be quickly detected and let aspects regarding the quality of the service being provided for mothers and the newborn to be evaluated. Specific interventions can also be programmed.

Key Words: Health surveillance, perinatal mortality, health indicator (source: $\mathrm{MeSH}, \mathrm{NLM}$ ).

$\mathrm{L}$ os indicadores en salud entendidos como: "una noción de la vigilancia en salud pública que define una medida de salud (la ocurrencia de una enfermedad o de otro evento relacionado con la salud) o de un factor asociado con la salud (el estado de salud u otro factor de riesgo) en una población especificada" (1), incluyen en su análisis, además de las medidas de morbilidad y mortalidad, otros aspectos como: el acceso a los servicios, la calidad en la atención, las condiciones de vida y los factores ambientales.

Entre los indicadores seleccionados por la OMS para la vigilancia del proceso reproductivo se encuentra la mortalidad perinatal: "muerte del producto que alcanza un mínimo de 1000 gramos o 28 semanas de gestación y hasta los siete primeros días de vida" (2), indicador de impacto, que mide en forma directa el estado de salud perinatal y, en forma indirecta, el estado de salud materna, y cuyo registro periódico, vigilancia y su posterior análisis, permiten programar intervenciones específicas y evaluar aspectos de la calidad de la atención.

Cartas de control estadístico de procesos

Entre las herramientas de control estadístico se encuentran las cartas de control estadístico de procesos (SPC), las cuales fueron desarrolladas en la segunda década del siglo veinte por Walter Shewart y difundidas en el mundo por W. Edwards Deming (3). Ellos observaron que las variaciones de un proceso pueden ser descritas por una distribución estadística, la cual en el área del cuidado de la salud puede ser del tipo: Poisson, binomial o geométrica (4-6).

La variabilidad en la calidad de un producto puede ser debida a causas aleatorias, sobre las cuales poco o nada se puede hacer o, a "causas especiales" o "causas asignables" sobre las que se puede tener algún grado de control. Una de las ventajas de las cartas de control es su capacidad para diferenciar las causas asignables de las no asignables (7), permitiendo un 
mejoramiento continuo al combinar el rigor de los métodos estadísticos clásicos (la significancia estadística) con el análisis cronológico de gráficas.

Los principios fundamentales de las cartas de SPC son (3):

Hay variaciones en las medidas individuales de procesos.

En datos provienen de un proceso con causas comunes y estables, su variabilidad es predecible dentro de un rango conocido que puede ser calculado por un modelo estadístico como la normal, la binomial o la distribución Poisson.

En un proceso que produce datos con causas especiales, las medidas se comportan de alguna forma específica observable, diferente de las distribuciones hipotéticas.

Asumiendo que los datos se encuentran bajo control, se pueden es tablecer las pruebas y los límites estadísticos para las desviaciones de las predicciones, suministrando evidencia estadística de los cambios.

Las cartas de SPC se clasifican de acuerdo al tipo de la característica de calidad monitoreada. Cartas de Control por Variables, se lleva el registro real de una característica de la calidad, son más sensibles y alertan rápidamente sobre problemas de calidad. Cartas de Control por Atributos, no hay medición continua sobre una característica de calidad y sólo se desea mostrar si el artículo se «adapta a la norma», evitan costosos procedimientos de medición y son fácilmente entendibles (8).

Una carta de control esta compuesta por dos partes: la primera parte consiste en una serie de puntos (medidas) graficados en orden cronológico y, la segunda (la carta de control), esta formada por tres líneas horizontales llamadas: la línea central, que generalmente es la media, el límite superior de control y el límite inferior de control. Para elaborarla se debe:

1. Elegir el plan de muestreo a utilizar.

2. Determinar el estadístico a ser graficado.

3. Calcular el estadístico para cada muestra.

4. Calcular el valor estimado para la distribución muestral del estadístico seleccionado.

5. Dibujar una línea horizontal en este valor, línea central.

6. Estimar la desviación estándar de la distribución muestral del estadístico seleccionado. Dibujar líneas horizontales a tres desviaciones estándar de la línea central, límite superior de control y límite inferior de control, respectivamente. 
Los límites de especificación no se deben colocar en la carta de control. Las especificaciones usualmente representan el mínimo de lo que es deseado y corresponden a los valores individuales, en cambio, los límites de control son para los promedios e intentan estimar lo que es actualmente posible. No hay conexión entre ellos (9).

Las técnicas estadísticas tradicionales en la literatura médica toman 2 SD como criterio estadístico para tomar decisiones pero hay importantes razones por las cuales las cartas control usan $\pm 3 \mathrm{SD}$. En un proceso estable, bajo control y con distribución normal, los límites de control estarán colocados a \pm 2 SD y el error tipo I (falsos positivos) para cada uno de los valores graficados podría estar alrededor del $5 \%$, valor muy alto comparado con $0,27 \%$ para una carta con los límites a $\pm 3 \mathrm{SD}$. En general, si un proceso con un tipo de distribución permanece bajo control, casi todos los datos seguirán cayendo entre \pm 3 SD de la media (3).

Se dice que los puntos están fuera de control cuando: un punto fuera de los límites de control, dos puntos fuera o tres puntos sucesivos a más de 2 desviaciones estándar de la media sobre el mismo lado de la línea central, cuatro puntos fuera o cinco puntos sucesivos a más de $1 \mathrm{SD}$ de la media sobre el mismo lado de la línea central, ocho puntos sucesivos sobre el mismo lado de la línea central, seis puntos sucesivos con tendencia creciente o decreciente o cuando hay comportamiento cíclico (3).

El objetivo de este trabajo fue aplicar la metodología de las cartas SPC, herramienta del control estadístico de la calidad, en la vigilancia de la mortalidad perinatal, indicador de la salud materno perinatal.

\section{MÉTODOS}

Las cartas de control SPC fueron elaboradas teniendo en cuenta 286 muertes perinatales reportadas a la Coordinación de Promoción y Prevención de la Empresa Promotora de Salud SUSALUD, al igual que los 51840 nacimientos, ocurridos entre enero de 2004 y diciembre de 2007.

La clasificación de muerte perinatal fue realizada por el Comité de Muertes Perinatales de la Empresa Promotora de Salud una vez analizada la historia clínica de la materna o del recién nacido, de acuerdo a los parámetros establecidos por el Centro Latinoamericano de Perinatología (CLAP). Inicialmente, la base de datos fue elaborada en Excel 2003 y la tabulación y el 
procesamiento de la información se realizó utilizando el paquete estadístico $\mathrm{R}(10)$.

Se elaboraron dos cartas de control: La primera carta se hizo teniendo en cuenta como línea central la proporción de muertes perinatales en cada uno de los meses y como límites superior e inferior de control la proporción de muertes $\pm 3 \mathrm{SD}$. La segunda, se elaboró calculando los logits de las proporciones de muertes perinatales, esto se hizo debido a que en el área de epidemiología es más natural expresar los riesgos en términos de Odds (cocientes de probabilidad) y a que desde el punto de vista teórico, el log del Odds converge a la normal más rápidamente (11).

\section{RESULTADOS}

La primera carta de control a considerar (Figura 1) se construyó así:

Figura 1. Carta control para la proporción de muertes perinatales, sin puntos fuera de control

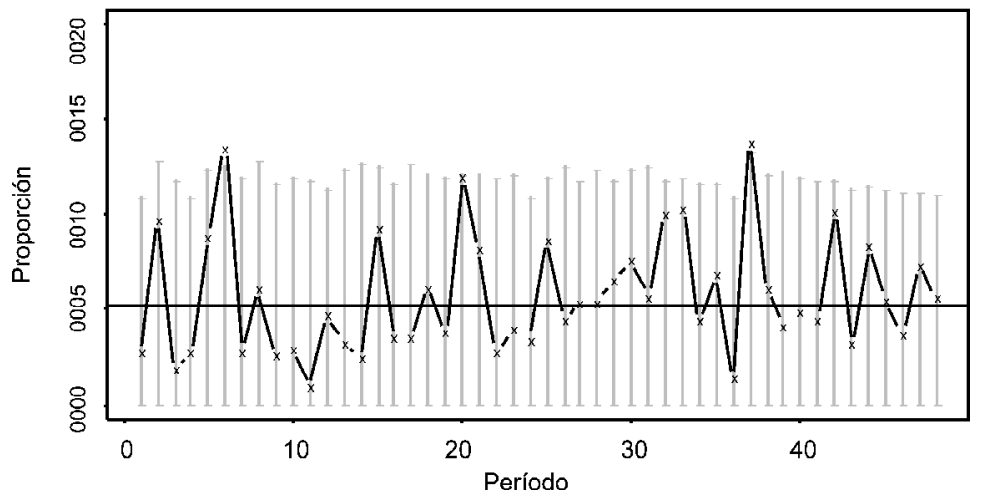

Fuente: Compañía Suramericana de Servicios de Salud S.A. Enero 2004 a diciembre de 2007.

1. Calculamos $\mathrm{p}=$ (total de muertes perinatales)/(total de partos) considerando todos los datos.

2. Calculamos LS y LI (provisionales) como:

$$
\begin{aligned}
& \mathrm{LS}_{\mathrm{i}}=\mathrm{p}+3 * \sqrt{ }\left(\mathrm{p}(1-\mathrm{p}) / \mathrm{n}_{\mathrm{i}}\right) \\
& \mathrm{LI}_{\mathrm{i}}=\mathrm{p}-3 * \sqrt{ }\left(\mathrm{p}(1-\mathrm{p}) / \mathrm{n}_{\mathrm{i}}\right)
\end{aligned}
$$

3. Estimamos las proporciones para cada uno de los meses, digamos $\mathrm{p}_{\mathrm{i}}=$ (muertes perinatales en el mes i)/(Número de partos en el mes i). Si algún punto (proporción) para un mes estaba por fuera de los límites se excluyó y se repi- 
tieron los pasos 1 y 2 nuevamente sin esta observación. Se obtuvo un promedio para el proceso igual a: $\mathrm{p}=0,005256451$ esto es, cinco muertes perinatales por cada mil nacimientos.

En esta carta los límites son variables ya que para cada mes se tiene un número diferente de partos. Se observa que los límites inferiores son todos cero, esto se debe a que los límites inferiores calculados fueron negativos. En este proceso se observan dos puntos «fuera de control» que corresponden a las observaciones 6 y 37 .

Si deseamos construir la carta usando las tasas entonces debemos recurrir al hecho que: si una variable aleatoria $X$ tiene media $\mu$ varianza $\sigma^{2}$, entonces $\mathrm{Y}=\mathrm{kX}$ ( $\mathrm{k}$ constante) tiene media $\mathrm{k} \mu$ y varianza $\mathrm{k}^{2 *} \sigma^{2}$. La carta resultante es idéntica a la anterior excepto en los límites del eje vertical.

Otra posibilidad que nos lleva a distintas conclusiones sería una carta de control para los logits (Figura 2).

Figura 2. Carta control para el Logit de muertes perinatales, sin puntos fuera de control

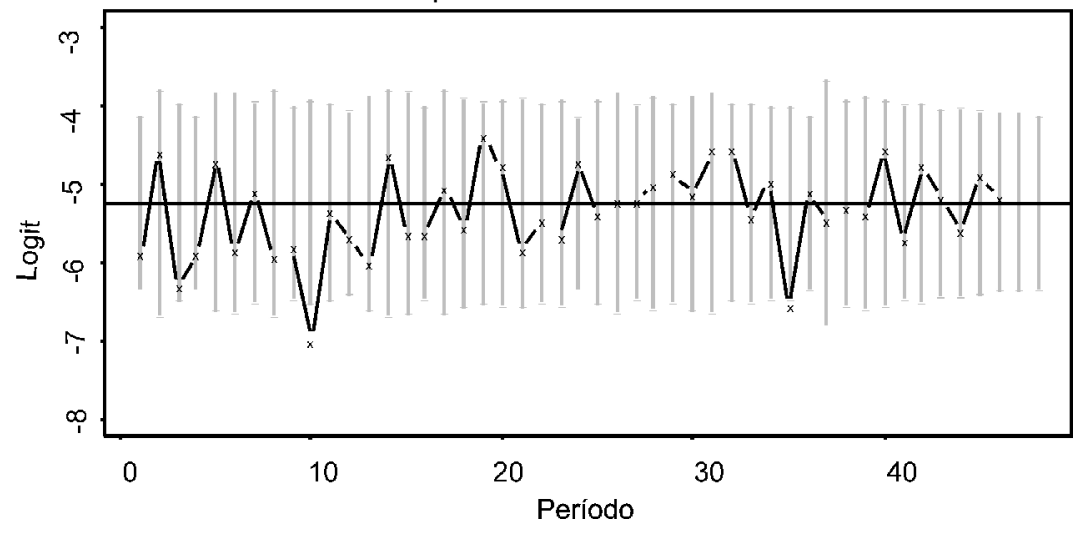

Fuente: Compañía Suramericana de Servicios de Salud S.A. Enero 2004 a diciembre de 2007.

El logit se define como $\log (\pi /(1-\pi))$. La distribución asintótica del log $\left(\pi^{\wedge} /\left(1-\pi^{\wedge}\right)\right)$ es normal con media $\log (\pi /(1-\pi))$ y varianza $1 /\left(\mathrm{n}^{*} \mathrm{p} *(1-\pi)\right)$

Esta carta señaló los puntos 11 y 36 como fuera de control que corresponden a las probabilidades observadas de 0,0009057971 y 0,001375516 , 
respectivamente, siendo las probabilidades más bajas. Si podemos determinar las causas asignables se podría mejorar el proceso como un todo.

\section{DISCUSIÓN}

La mortalidad perinatal es un indicador de impacto, que refleja en forma directa, la atención prenatal, intraparto y neonatal y, en forma indirecta, la salud materna. Dado que la muerte perinatal es un evento más frecuente que la muerte materna, puede constituirse en un indicador más sensible del estado de salud reproductiva, incluida la salud materna, y sirve por lo tanto como un indicador de la calidad del servicio de salud materno-infantil. Las tasas decrecientes a través del tiempo son el reflejo de un buen estado de salud pero, las tasas crecientes pueden reflejar un verdadero deterioro de la calidad de los servicios o del acceso a los mismos. Sin embargo, debe tenerse en cuenta que un mejor conteo y registro de las defunciones fetales también pueden producir un aumento aparente en la tasa.

En las últimas décadas, se han utilizado para la vigilancia de eventos en salud los corredores o canales endémicos (12), los cuales son construidos teniendo en cuenta una medida de tendencia central y su recorrido. Una de las medidas más utilizadas actualmente en su construcción es la media geométrica y su intervalo de confianza pero, para utilizarla, es necesario que todos los valores sean mayores que cero, lo cual no es posible en el caso de la mortalidad perinatal pues este indicador para algunos periodos puede ser igual a este valor.

Las cartas de control estadístico de procesos, son cada vez más utilizadas para orientar el análisis de los indicadores utilizados en el monitoreo de los procesos clínicos y sus resultados. Ellas ayudan a generar hipótesis acerca de las causas de la variación dirigiendo los esfuerzos hacia las acciones más eficientes con las que se logre mejorar la calidad en la atención.

Este trabajo permitió elaborar dos cartas de control para el monitoreo de la mortalidad perinatal, uno de los indicadores del proceso reproductivo, las cuales pueden ser usadas para vigilar la variación del mismo a lo largo del tiempo y probar la efectividad de las acciones correctivas emprendidas. La primera carta control se elaboró teniendo en cuenta las proporciones de muertes perinatales para cada uno de los meses y, la segunda, los logits de las proporciones de muertes perinatales, los cuales desde el punto de vista teórico, convergen más rápidamente a la normal. 
Otra carta de control alternativa hubiera podido ser, en este caso, la carta Poisson, ya que, como es sabido, la distribución Poisson es una distribución límite para la distribución binomial, pero consideramos que era más apropiado trabajar con la distribución exacta, la que, por otra parte, considera los tamaños muestrales diferentes

Agradecimientos. Agradecemos a la Compañía Suramericana de Servicios de Salud S.A. SUSALUD, por permitir la utilización de la base de datos con los cuales fue realizado este trabajo y autorizar la publicación de los resultados.

\section{REFERENCIAS}

1. Organización Panamericana de la Salud. Indicadores de Salud: Elementos Básicos para el Análisis de la Situación de Salud Boletín Epidemiológico 2001; 22(4): 1-5.

2. Mortalidad Materna y Perinatal. Protocolo de Vigilancia Epidemiológica para la Mortalidad Materna y perinatal [Internet]. Dirección Seccional de Salud de Antioquia Disponible en: http:// www.dssa.gov.co/dowload/Protocolos/P008.pdf. Consultado Abril de 2008.

3. Benneyan JC, Lloyd RC, Plsek PE. Statistical process control as a tool for research and healdcare improvement. Qual Saf Health Care 2003; 12: 458-464.

4. Benneyan JC, Borgman AD. Risk-adjusted sequential probability ratio tests and longitudinal surveillance methods. International Journal for Quality in Health Case 2003; 15(1): 5-6.

5. Marchall T, Mohammed MA. Case-mix and the use of control charts in monitoring mortality rates after coronary artery bypass. BMC Health Services Research 2007; 7:63.

6. Harrison WN, Mohammed MA, Wall MK, Marchall T. Analysis of inadecuate cervical smears using Shewhart control charts. BMC Public Health 2004; 4:25.

7. Poco A, Farias S. [Internet]. El control estadístico de procesos mediante las cartas de control. Disponible en: http://www.fceco.uner.edu.ar/epn/catedras/matenmat/a11pf.doc. Consultado Marzo de 2008.

8. Mustafa AM, Rodríguez NL, Chauvet S. [Internet]. Control de Calidad: Cartas de Control por variables. Congreso Regional de Ciencia y Tecnología NOA 2002. Secretaría de Ciencia y Tecnología. Universidad Nacional de Catamarca. Disponible en: http:// www.editorial.unca.edu.ar/NOA2002/Control\%20Calidad\%20Cartas.pdf. Consultado Marzo de 2008

9. Grant EL, Leavenworth RS. Control Estadístico de Calidad. México: C.E.C.S.A; 1977. p. 327-352.

10. Development Core Team. Foundation for Statistical Computing [Internet]. A Language and Environment for Statistical Computing, Versión 2.7.0, Vienna, Austria; 2008. Disponible en: http://www.R-project.org. Consultado Marzo de 2008.

11. Serfling RJ. Approximations Theorems of Mathematical Statistics. New York: John Wiley \& Sons; 1980.

12. Bortman M. Elaboración de corredores o canales endémicos mediante planillas de cálculo. Rev Panam Salud Pública 1999; 5(1): 1-8. 